\title{
Agitating cylindrical particles in laminar liquid flow
}

\author{
J.J. Derksen \\ School of Engineering, University of Aberdeen, Aberdeen, UK \\ jderksen@abdn.ac.uk
}

Submitted to Chemical Engineering Research \& Design - August 2019

Revision submitted: November 2019

Accepted: December 2019

\begin{abstract}
Three-dimensional, time-dependent simulations of dense agitated solid-liquid suspensions involving particles of cylindrical shape in a Newtonian liquid have been performed. The liquid flow is resolved by the lattice-Boltzmann method at length scales finer than the size of the particles, which implies particleresolved simulations. The flow solution includes the hydrodynamic forces and moments on each particle that are used to integrate their linear and rotational equations of motion. No-slip at the particle surfaces is imposed by an immersed boundary method (IBM). The marker points of the IBM are also used to detect and carry out collisions between particles. This numerical procedure has been applied to systems contained in a rectangular box and agitated by a revolving disk as well as by a pitched-blade turbine with an impeller-based Reynolds number of 87 , which indicates laminar flow. The overall solids volume fraction has been fixed to $15 \%$; the number of particles is of the order of one thousand. We study the effect of impeller type and particle shape (in terms of the length over diameter ratio of the cylinders that has been varied between 1 and 4) on the extent to which the solids are suspended and on the way the cylinders orient themselves.
\end{abstract}

\section{Keywords}


Solids suspension, particle-resolved simulations, non-spherical particles, lattice-Boltzmann method, agitated flow. 


\section{Introduction}

There are numerous challenges when it comes to predictive simulations of liquid flows carrying solid particles, specifically for industrially relevant solid-liquid systems. To name some important ones: resolving turbulence in the fluid phase and accurately accounting for how flow turbulence interacts with the particulate phase; representing (wide) particle size distributions; dealing with dense, collisiondominated suspensions and accounting for the shape of the particles. This paper focuses on the latter two aspects: dense suspension of non-spherical particles. At the same time, it does not consider turbulence and size distributions. The reason for not considering turbulence at this stage is that we aim for simulations that accurately represent the shape of particles as well as the close-range interactions between particles in dense suspensions, i.e. we aim for particle-resolved simulations. This needs three-dimensional grids that are finer (by at least one order of magnitude in each coordinate direction) than the particle size so that we need to restrict ourselves to small systems that are hardly able to develop turbulence.

Computational research on solid particles suspended in liquids has so far largely focused on particles of spherical shape. For example, models for solid-liquid interaction are usually formulated in terms of drag force correlations for spheres [Khandai et al 2003; Van der Hoef et al 2005; Tenneti et al 2011]; constitutive models for solids derived from kinetic theory of granular matter normally assume the granular equivalent of monoatomic gases, which implies spherical particles [Gidaspow 1994]; and also particle-resolved, direct simulations mostly involve spheres [Kidanemariam \& Uhlmann 2017; Vowinckel et al 2014; Derksen \& Sundaresan 2007]. Particle shape, however, is expected to have significant impact on the individual behavior of particles and therefore also on the collective behavior of solids-liquid mixtures [Richardson \& Zaki 1954]. In order to elucidate particle-shape effects in a computational manner, we need a simulation procedure that explicitly accounts for the shape of the particles.

With this in mind we have devised a method for particle-resolved simulations of dense solid-liquid suspensions involving non-spherical particles [Shardt \& Derksen 2012; Derksen 2019]. Given the high solids volume fractions we are interested in, collision handling and close-range interactions between 
particles are an important feature of the numerical procedure. So far, we have applied this method to fully periodic systems and studied solid-liquid fluidization with cylindrical particles as well as settling of solid particles having the shape of red blood cells. These are homogeneous systems without walls. In order to increase the flow's complexity, to study interactions with walls and moving solid objects (such as a revolving impeller), and also to simulate flow systems that can be easily replicated experimentally, we here report the behavior of solid-liquid suspensions with non-spherical particles in small agitated tanks. As we will see, these are strongly inhomogeneous and often only partially suspended systems. In such cases, part of the particles form a granular bed on the bottom of the container. Mobilizing the bed critically depends on how the bed is packed with the structure of the packing strongly influenced by the shape of the particles. Granular bed erosion has implications well beyond agitated tanks in areas such as sediment transport in pipelines, rivers, and coastal regions [Uijttewaal 2014; Ramesh et al 2011]. One advantage of working with small, confined agitated systems is that they can be built easily and are amenable to quantitative (flow) visualization experiments, ideally with refractive index matching between solids and liquid so that one can look deep inside the system. As an example, we have reported refractiveindex-matched Particle Image Velocimetry (PIV) experiments for measuring flow velocities of the interstitial liquid in an agitated solids suspension involving spherical particles [Li et al 2018].

The aim of this paper is to study the way particles of cylindrical shape are entrained by laminar liquid flow and how this depends on the way the liquid is agitated, and on the shape (here aspect ratio, length over diameter) of the particles. From a computational methods perspective, we show in this paper that the immersed boundary method is an elegant way of not only dealing with imposing no-slip boundary conditions on moving solid surfaces but is also instrumental when it comes to collision detection and handling between particles and between particles and a revolving impeller.

This paper is organized in the following manner: First the flow geometries and conditions are defined, mostly in dimensionless terms. We then briefly describe the numerical procedure which compared to previous papers [Shardt \& Derksen 2012; Derksen 2019] - has been extended with particle- 
wall and particle-impeller interactions. Subsequently parameter settings and parameter ranges are discussed. In the Results section we begin by qualitatively showing particle suspension levels for different mixing configurations. These observations are then quantified in terms of the average elevation of particles as a function of process conditions as well as how solids and their kinetic energy are distributed over the height of the tank under dynamically steady conditions. Also the orientation of the particles and their alignment with the flow field have been investigated. The final section presents conclusions and suggestions for future work.

\section{Flow systems}

The three-dimensional flow domain is a rectangular box closed on all sides with a square footprint $T \times T$ and a height $H=0.96 T$. It contains a Newtonian liquid with density $\rho$ and kinematic viscosity $\nu$, as well as $n$ cylindrically shaped solid particles with length $\ell$, diameter $d$, and density $\rho_{p}>\rho$. The solidsliquid mixture is agitated by spinning an impeller with diameter $D=0.53 T$ at a rate of $N$ revolutions per unit time placed in the center of the flow domain (see Figure 1 that also defines the Cartesian coordinate system used throughout this paper). Two different impellers that have the same diameter $D$ have been used: a circular disk and a $45^{\circ}$ pitched-blade turbine (PBT) with four impeller blades that pump in the downward direction; geometrical details are given in Figure 1. Gravity acts in the negative $z$-direction: $\mathbf{g}=-g \mathbf{e}_{\mathbf{z}}$

The parameters introduced above have been combined in a set of dimensionless numbers: the impeller-based Reynolds number $\operatorname{Re}=N D^{2} / \nu$ is an important characteristic of the liquid flow; the Shields number quantifies the inertial forces suspending a particle relative to net gravity pulling the particle down $\theta=\rho N^{2} D^{2} /(g \Delta \rho d)$ with $\Delta \rho=\rho_{p}-\rho$; the density ratio is $\gamma=\rho_{p} / \rho$; and the overall solids volume fraction is $\langle\phi\rangle=\frac{n \pi \ell d^{2}}{4 H T^{2}}$. The cylindrical particles have an aspect ratio $\ell / d$. To quantify the size of a particle relative to the stirring system we take $d / D$. 
The above set of dimensionless groups fully defines the flow system in a physical sense. Other dimensionless numbers can be formed by combining the above groups. As an example, previously [Derksen 2019] we identified the Archimedes number based on the equivalent diameter $\operatorname{Ar}_{e}=(\gamma-1) g d_{e}^{3} / \nu^{2}$ (with $\left.d_{e}=\sqrt[3]{3 \ell d^{2} / 2}\right)$ as a useful parameter for scaling the hindered settling speed for particles with different aspect ratio $\ell / d$. This Archimedes number can be formed by combining Re, $\theta$ and aspect ratios.

\section{Simulation procedure}

At the heart of the numerical simulations presented here is the lattice-Boltzmann (LB) method [Chen \& Doolen 1998; Aidun \& Clausen 2010]. It solves - in a time-dependent manner - the continuity and Navier-Stokes equations in three dimensions in the low Mach number limit on a uniform, cubic grid with spacing $\Delta$. It has been supplemented with an immersed boundary method (IBM) to impose no-slip at solid surfaces moving through the flow domain [Ten Cate et al 2002]. In our simulations these solid surfaces belong to the impeller as well as to the particles. In the simulations, the solid surfaces have been defined by sets of closely spaced marker points. The distance between neighboring marker points is typically in the range $0.5-0.75 \Delta$. At these - off-grid - marker points we determine, through interpolation from the grid, the fluid velocity and subsequently the local velocity difference between fluid and solid. We then locally apply forces on the fluid to bring this velocity difference to zero and in this way impose the no-slip condition. The IBM (combined with the LB method) has been validated experimentally for spherical particles [Ten Cate et al 2002]; its convergence rate is first order.

For solid surfaces with prescribed velocity - such as the impeller - this method has been introduced in a LB context in [Derksen \& Van den Akker 1999]. For the surfaces of the freely moving particles, the local forces required for no-slip are integrated over the particle surface to determine the overall hydrodynamic force and torque on the particle that are then used to update its linear and angular velocity as well as its location and orientation. For spherical particles, that do not require orientation updates, this 
procedure has been discussed in [Ten Cate et al 2002; Derksen \& Sundaresan 2007]. For non-spherical particles we use quaternions for keeping track of the orientation of the particles [Kuipers 1999]. This has been described in more recent papers of ours [Shardt \& Derksen 2012; Derksen 2019]. The outer bounding - walls of the container are aligned with the cubic grid. Here no-slip is imposed by applying the half-way bounce-back rule on the LB distribution functions [Succi 2001].

We are dealing with three types of collisions: particle-particle, particle-impeller, and particle-wall (where "wall" stands for the fixed, bounding walls of the container). Collisions are a challenging part of the simulation process. Where collisions between spherical particles can be easily anticipated by monitoring the distance between center locations of nearby particles, this is less straightforward for collisions involving non-spherical particles. In the method we have proposed [Derksen 2019], the marker points used in the IBM are also used to detect proximity of solid particle surfaces. When the proximity falls below a certain threshold, this locally activates an elastic repulsive force between the particles that prevents them from overlapping. Next to the location of the marker points, also the outward normal at that location has been stored so that the direction of the repulsive force can be determined. In addition to an elastic repulsive force, a damping force proportional to the relative velocity of nearby marker points on two different particles is calculated. This damping accounts for unresolved lubrication forces [Kim \& Karrila 1991; Nguyen \& Ladd 2002]. The above procedure has been explained in detail for particleparticle collisions [Derksen 2019], including the choice of parameters (spring and damper constants, cutoff and saturation distances) involved. For completeness and reference, the expressions for elastic and damping forces are given in Table 1, along with the values of the parameters used in the current study. Note that the parameters are given in lattice units: unit of length is the grid spacing $\Delta$, unit if time is the time step $\Delta t$, unit of mass is the average mass per $\Delta^{3}$ (cubic) lattice cell.

In our previous paper [Derksen 2019], cylindrically shaped solid particles moved through fully periodic domains with the flow driven by gravity (down) and a pressure gradient (up) and we had no agitation device and no bounding walls. In the current paper, the flow is agitated by a revolving impeller. 
The extension towards particle-impeller collisions is relatively straightforward since also the impeller is represented by means of the immersed boundary method. The marker points on the impeller have outward normal vectors associated to them and exactly the same procedure as applied for particle-particle collisions has been adopted for particle-impeller collisions, including the values of the collision parameters: If the distance between a marker point on a particle and a marker point on the impeller falls below the threshold distance an elastic repulsive force and a damping force are activated. The latter depends on the relative velocity between the marker point on the impeller and the one on the particle.

For particle-wall collisions the proximity of marker points and bounding walls is monitored. As for the other collision types, when the distance between a marker point on a particle and a wall gets smaller than the threshold distance, forces on the particle are activated that effectively perform the particle-wall collision in the same way as particle-particle and particle impeller collisions. The elastic part of the collision force acts in the wall-normal direction.

\section{Set-up of the simulations}

The $T \times T \times H$ flow domain is discretized with $n x \times n y \times n z$ cubic cells with $n x=n y=251$ and $n z=240$. In this domain, the impeller is represented by a collection of marker points and associated outward unit normal vectors at its surface. The two impellers (disk and PBT) both have a diameter that spans $D=132 \Delta$ lattice spacings. The marker points revolve around the $z$-axis such that the impeller makes one revolution in 4000 time steps $(N=1 /(4000 \Delta t))$. In all simulations the Reynolds number has the fixed to a value of $\operatorname{Re}=87$. This is achieved by setting the kinematic viscosity of the liquid to $\nu=0.05$ in lattice-units. It therefore is important to note that we are dealing with laminar liquid flow.

The particles are of cylindrical shape and have a diameter of 12 lattice spacings $(d=12 \Delta)$. In this paper, particles with three aspect ratios have been investigated: $\ell / d=1,2$, and 4 . In our previous paper [Derksen 2019], a grid-refinement study has been performed. Results with resolutions of $d=12 \Delta, 16 \Delta$ and $24 \Delta$ for dense liquid-fluidized suspensions were compared in terms of average and fluctuating 
velocities. Reasonable agreement between the different resolutions was observed. As we will see, we need many impeller revolutions to develop the two-phase flow systems to a dynamically steady state. Therefore, with a view to computational affordability, in this paper we have chosen the lower resolution of $d=12 \Delta$.

As mentioned above, Table 1 shows the settings of the collision parameters, along with the equations for the normal elastic collision force, the normal lubrication force and the tangential lubrication force. These settings are the same as used in [Derksen 2019]. For particle-impeller and particle-wall collisions the same parameters as for particle-particle collisions have been used.

For initialization of the simulations we first randomly distribute the particles in a non-overlapping way in the $T \times T \times H$ flow domain, without the impeller being present. We let the particles settle so that they eventually form loosely packed random beds on the bottom. Impressions of these beds are given in Figure 2. We then insert the impeller. Only situations have been considered with all the particles initially underneath the impeller. In the first 4000 (i.e. 1/ $N$ ) time steps, the impeller speed linearly increases to its steady-state value so that in these initial 4000 time steps only half an impeller revolution is completed. Beyond the first 4000 time steps, the impeller rotates with a constant angular velocity. The solid-liquid flow system is monitored so as to estimate when it reaches a quasi-steady state. After steady state is reached we continue the simulation in order to collect statistical flow data.

A set of six simulations involving all combinations of the two impellers and the three particle aspect ratios will be discussed. In addition to fixing the Reynolds number to $\mathrm{Re}=87$, also the density ratio $\gamma=\rho_{p} / \rho=1.25$, the ratio of particle size over impeller diameter (at $d / D=0.091$ ), as well as the overall solids volume fraction $(\langle\phi\rangle=0.148)$ have fixed values. The latter means $n=1644$ in case $\ell / d=1, n=822$ if $\ell / d=2$, and $n=411$ if $\ell / d=4$ with $n$ the total number of particles in the container.

The emphasis in analyzing the results will be on comparing how particles with different aspect ratio respond to the flow induced by the disk and by the PBT. All particles are made of the same material; in experimental terms this means that we cut them with different length from long rods of diameter $d$ and 
expose them to fluid flow. We thus compare the behavior of particles with different aspect ratio at the same Shields number $\theta=\rho N^{2} D^{2} /(g \Delta \rho d)=9.08$.

\section{Results}

Impressions of the solids-liquid flow generated by the spinning disk are given in Figure 3 . The disk creates a swirling flow along with a much weaker radial flow coming off the disk. This radial stream recirculates in the container [Mo et al 2015]. The swirling flow creates a low-pressure zone near the center of the tank. Since the swirl gets weaker further away from the disk, a negative vertical pressure gradient $(\partial p / \partial z<0)$ develops on the centerline underneath the disk [Mo et al 2015]. This provides a driving force for upward vertical motion of the particles in the granular bed. This lifting of the particles in the upper layers of the bed underneath the impeller locally dilutes the bed and makes that the mostly horizontal swirling shear flow can penetrate the bed and move the particles, also in the horizontal direction. The collisions between particles that are the result of this motion - particles tumbling over one another - helps in the entrainment of the solids in the liquid stream. As can be judged from the snapshots in Figure 3, the suspension process is slightly faster for the larger $\ell / d=4$ cylinders compared to the $\ell / d=1$ cylinders, despite that the net weight of the former is 4 times larger. Compared to the disk, the PBT creates a stronger flow that has larger vertical velocity components which leads to a faster suspension process, see Figure 4. Also with a PBT the longer cylinders get suspended better than the shorter ones.

Time series of the average vertical center location of the particles $\left\langle z_{p}\right\rangle$ are presented in Figure 5 .

For the disk (upper panel of Figure 5) we follow $\left\langle z_{p}\right\rangle$ from the moment $t=0$ when all particles are resting on the bottom and on one another and the disk is set to rotate. In the time series related to the PBT we have omitted some of the first stages of the suspension process and - instead - focus on the two-phase system reaching a dynamic steady state. We note that we need long simulations, covering more than 100 
impeller revolutions to develop the systems to steady state (and more than 200 revolutions for the combination PBT and $\ell / d=1)$. In all cases, steady state values of $\left\langle z_{p}\right\rangle / H$ are well below 0.5 . Since a uniform distribution of particles over the tank volume would result in $\left\langle z_{p}\right\rangle / H=0.5$, it is clear that under the given conditions - the impellers are only able to partially suspend the solid cylinders. For the disk there is a small but significant difference in the steady value for $\left\langle z_{p}\right\rangle / H$ with different $\ell / d:$ the longer the cylinders, the higher $\left\langle z_{p}\right\rangle / H$. The PBT is spinning with the same speed as the disk, agitating the same solids-liquid system, and is able to better suspend the particles. The differences between the suspension levels of the different length cylinders is more pronounced than for the disk with steady state values of $\left\langle z_{p}\right\rangle / H$ of $0.37,0.31,0.26$ for $\ell / d=4,2,1$ respectively.

In Figure 6, suspension levels are further examined in the form of time-averaged vertical solids volume fraction profiles where time averaging has been performed over a time window of a duration of 10 impeller revolutions after the flow has reached dynamic steady state. The resolution of the profiles is finer than the diameter of the cylinders. This makes the layering of the particles on the bottom visible in the profiles. It is noted that the layering is more pronounced for the PBT as compared to the disk.

That the disk shows lower $\left\langle z_{p}\right\rangle / H$ levels than the PBT is largely due to the fact that the solids hardly reach vertical levels higher than the disk: the disk is an obstacle for vertical mobility of the particles. Solids concentrations above the PBT are appreciable, specifically for the $\ell / d=4$ cylinders.

The dynamics of the particles we quantify in terms of their kinetic energy and the way it is distributed over the tank volume, specifically in the vertical direction. The kinetic energy associated to linear motion is $k_{\text {lin }}=\frac{1}{2} m_{p}\left|\mathbf{u}_{\mathbf{p}}\right|^{2}$ with $m_{p}$ the particle mass and $\mathbf{u}_{\mathbf{p}}$ its velocity respectively; for the rotational motion it is $k_{r o t}=\frac{1}{2}\left(I_{1} \omega_{p 1}^{2}+I_{2} \omega_{p 2}^{2}+I_{3} \omega_{p 3}^{2}\right)$ with $1,2,3$ denoting the principal axes of the cylinders (see e.g. [Derksen 2019]) and $I_{i}(i=1,2,3)$ and $\omega_{p i}(i=1,2,3)$ the moments of inertia and 
angular velocities along these axes respectively. Non-dimensional profiles of kinetic energy are given in Figure 7. They show extreme concentration for the flow driven by the disk, just underneath the disk. The virtual absence of particles above the disk leads to negligible solids kinetic energy levels there. Overall for the disk, the energy contained in the linear motion is at least an order of magnitude larger than in the rotational motion of the particles. We also observe that the longer cylinders store more energy in rotation than the shorter ones.

Wider vertical kinetic energy distributions are observed for the flows driven by the PBT. This is due to the solids being more evenly distributed over the height of the container. Different from the disk, the kinetic energy contained in linear motion strongly depends on the aspect ratio of the particles. This is a reflection of the longer particles being better suspended than the shorter ones. The kinetic energy contained in rotational particle motion shows a vertical distribution very similar to that of linear motion. Its values are, however, lower by more than one order of magnitude.

For non-spherical particles it is relevant to monitor their orientation. For this purpose the angle $\varphi$ is defined as the angle between the cylinder's center line and the vertical direction. The impression of the long $(\ell / d=4)$ cylinders in Figures 3 and 4 is that they mainly orient horizontally so that we expect the distribution of angles $\varphi$ to be skewed towards $\varphi=\pi / 2$. Figure 8 shows that this is indeed the case for $\ell / d=4$ as well as for $\ell / d=1$. The angle distribution for $\ell / d=1$ also shows a peak near $\varphi=0$. This relates to the short cylinders resting on the bottom on their end-plane. The peaks in the angle distributions are almost exclusively due to the unsuspended particles: If we exclude the particles with $z_{p} \leq 0.2 H$ from the angle distributions, the peaks disappear (see Figure 9). The choice for $z=0.2 H$ to distinguish between suspended (i.e. mobile) and unsuspended particles is based on the vertical solids volume fraction profiles in Figure 6 that show that layering only occurs beneath $z=0.2 \mathrm{H}$.

For reference we plot $\sin \varphi$ in Figure 8 (and also in the subsequent Figure 9). Randomly oriented cylinders with length $\ell$ will have their end points uniformly distributed over a sphere with diameter $\ell$. 
The vertical location of these endpoints will then be distributed according to $\frac{1}{2} \ell \sin \varphi$ and the angle $\varphi$ therefore is distributed according to $\sin \varphi$.

Orientation angle distributions of mobile particles are shown in Figure 9 . The short $\ell / d=1$ cylinders orient themselves largely in a random fashion, with only a slight preference for horizontal, $\varphi=\pi / 2$, orientation. The longer cylinders tend much more to a horizontal direction, most pronounced for the $\ell / d=4$ cylinders in the flow generated by the disk. In that situation cylinders align along the bottom surface of the disk.

As a more global flow characteristic we now consider the torque and - directly related - the power required to rotate the impeller. Given the two-phase nature of the flow system, one can distinguish between two contributions to the torque: in the first place the "fluid torque" which is due to the fluid stress distribution (including pressure) over the impeller surface, in the second place the "solids torque" that is the result of collisions between particles and the impeller surface. Both have been calculated explicitly in the simulation procedure: The fluid torque through the immersed boundary method that forces no-slip at the impeller surface; the solids torque through the contact forces (see Table 1) that handle particle-impeller collisions.

Results for the PBT are presented in Figure 10 in terms of the dimensionless power number Po $\equiv 2 \pi N \Gamma /\left(\rho N^{3} D^{5}\right)$ with $\Gamma$ the torque. In the first place we observe significant levels of fluctuations in Po, although we are dealing with laminar flow. The erratic motion of the particles is responsible for the fluctuations; in a direct way when it comes to the solids torque contribution but also in an indirect way via the fluid, i.e. the solids locally influencing fluid flow and therefore the fluid stress distribution over the impeller surface. This is confirmed by the power number measured in the absence of particles as displayed in the lower panel of Figure 10 (green curve). Its marginal fluctuations are periodic and relate to interaction between impeller blades and the tank walls. The better suspended $\ell / d=4$ particles thus produce stronger fluctuations and also an on average higher power number. In addition, the $\ell / d=4$ 
particles have the highest mass and thus the most inertia with direct consequences for the levels of solids torque.

Time averaged quantities for the power number are given in Table 2. The division over solids and fluid torque strongly depends on the aspect ratio and so does the overall power number. Power characteristics are important from a practical point of view as they are a means of - global - experimental validation of the results since torque is a measurable quantity.

\section{Summary and conclusions}

We have reported simulations on solids suspension in a container agitated by a disk and by a pitchedblade turbine (PBT) under laminar flow conditions with the particles having cylindrical shape. This particle shape is fully accounted for through the immersed boundary method that imposes no-slip at the cylinder surfaces and that also assists in handling collisions between particles, between particles and the impeller as well as between particles and the container wall. With the lattice-Boltzmann method as flow solver, the developed numerical procedure is capable of particle-resolved simulations with of the order of one thousand particles in complex flow - here the flow generated by revolving impellers - over appreciable process time (over one hundred impeller revolutions). It is worthwhile noting that the simulations reported here have been run in sequential mode; parallelization has the potential of performing simulations of larger systems and/or simulations with higher spatial and temporal resolution.

In the first instance we let the cylindrical particles settle in the container so that they form a loosely packed, random granular bed on the bottom. After switching on the agitation we keep track of the suspension process. It takes at least 100 impeller revolutions for the two-phase systems to develop a dynamic steady state. A relatively low inertial Shields number of 9.1 has been applied that results in partially suspended solids with the particles that remain on the bottom forming a layered structure. As is expected, the disk does not perform very well in mobilizing and suspending particles. Not only is the flow it generates relatively weak, it also forms a major obstruction for particles migrating upwards. The main 
mechanism for particle suspension by the disk is the vertical pressure gradient as a result of the swirling flow it creates. Particles are more effectively suspended by the PBT that creates a stronger fluid flow with more pronounced vertical components.

Since the particles are inhomogeneously distributed over the tank volume, this also is the case for the kinetic energy of the particles. The kinetic energy in the rotational motion of the particles is much less - by one order of magnitude - than that contained in the linear motion. The larger the $(\ell / d)$ aspect ratio of the particles, the more energy is in particle rotation. Also the orientation of the particles clearly depends on the aspect ratio with the longer particles tending to a horizontal orientation whereas the shorter particles are largely randomly oriented.

The simulations provide data for the torque required to spin the impeller. This is an interesting avenue for experimental validation in terms of this global parameter. It was shown that the torque has a contribution directly related to collisions between the particles with the impeller, and a contribution due to the liquid flow. The latter is - of course - strongly influenced by the presence of particles and for that reason fluctuates strongly, although the flow is laminar. The relative strength of the two contributions depends on the aspect ratio of the cylinders.

With a view to practical applications there is a need to increase the Reynolds numbers so as to create mildly turbulent flow in the mixing tank. This will likely require higher resolution, not only to resolve the flow generated by the impeller but also the flow around the cylindrical particles. First and foremost, however, it is important to establish experimental validation of the numerical procedure. For this reason we will be working on studying the behavior of a single cylinder and the way it gets suspended in laminar flow driven by a disk and then experimentally visualize multi-particle systems as the ones simulated in the current paper. 


\section{References}

Aidun, C. \& Clausen, J. (2010). Lattice-Boltzmann method for complex flows. Annual Review of Fluid Mechanics, 42, $439-472$.

Chen, S. \& Doolen, G.D. (1998). Lattice-Boltzmann method for fluid flows. Annual Review of Fluid Mechanics, 30, 329-364.

Derksen J. \& Van den Akker H.E.A. (1999). Large-eddy simulations on the flow driven by a Rushton turbine. AIChE Journal, 45, 209-221.

Derksen, J,J. \& Sundaresan S. (2007). Direct numerical simulations of dense suspensions: wave instabilities in liquid-fluidized beds. Journal of Fluid Mechanics, 587, 303-336.

Derksen, J.J. (2019). Liquid fluidization with cylindrical particles: Highly resolved simulations. AIChE Journal, 65, pe16594, 1-11, 2019.

Gidaspow D. (1994). Multiphase flow and fluidization. Academic Press, San Diego.

Khandai, D., Derksen, J.J. \& Van den Akker, H.E.A. (2003). Interphase drag coefficients in gas-solid flows. AIChE Journal, 49, 1060-1065.

Kidanemariam, A.G. \& Uhlmann, M. (2017). Formation of sediment patterns in channel flow: minimal unstable systems and their temporal evolution. Journal of Fluid Mechanics, 818, 716-743.

Kim S. \& Karrila S.J. (1991). Microhydrodynamics: Principles and selected applications. ButterworthHeinemann, Boston.

Kuipers J.B. (1999). Quaternions and Rotation Sequences. Princeton University Press, Princeton.

Li, G., Gao, Z., Li, Z., Wang, J. \& Derksen, J.J. (2018). Particle-resolved PIV experiments of solid-liquid mixing in a turbulent stirred tank. AIChE Journal, 64, 389-402.

Mo, J., Gao, Z., Li, Z., Bao, Y. \& Derksen, J.J. (2015). Suspending a solid sphere in laminar inertial liquid flow - experiments and simulations. AIChE Journal, 61, 1455-1469.

Nguyen, N-Q. \& Ladd, A.J.C. (2002). Lubrication corrections for lattice-Boltzmann simulations of particle suspensions. Physical Review E, 66, 046708.

Ramesh, B., Kothyari, U.C. \& Murugesan, K. (2011). Near-bed particle motion over transitionally-rough bed. Journal of Hydraulic Research, 49, 757-765.

Richardson, J.F. \& Zaki, W.N. (1954). Sedimentation and fluidisation. Part 1. Transactions of the Institute of Chemical Engineers, 32, 35-53. 
Shardt, O. \& Derksen, J.J. (2012). Direct simulations of dense suspensions of non-spherical particles. International Journal of Multiphase Flow, 47, 25-36.

Succi, S. (2001). The lattice Boltzmann equation: For fluid dynamics and beyond. Oxford University Press, Oxford.

Ten Cate, A., Nieuwstad, C.H., Derksen, J.J. \& Van den Akker HEA. (2002). PIV experiments and lattice-Boltzmann simulations on a single sphere settling under gravity. Physics of Fluids, 14, 40124025.

Tenneti, S., Garg, R. \& Subramaniam, S. (2011). Drag law for monodisperse gas-solid systems using particle-resolved direct numerical simulation of flow past fixed assemblies of spheres. International Journal of Multiphase Flow, 37, 1072-1092.

Uijttewaal, W.S.J. (2014). Hydrodynamics of shallow flows: application to rivers. Journal of Hydraulic Research, 52, 157-172.

Van der Hoef, M.A., Beetstra, R. \& Kuipers, J.A.M. (2005). Lattice-Boltzmann simulations of lowReynolds-number flow past mono- and bidisperse arrays of spheres: results for the permeability and drag force. Journal of Fluid Mechanics, 528, 233-254.

Vowinckel, B., Kempe, T. \& Fröhlich, J. (2014). Fluid-particle interaction in turbulent open channel flow with fully-resolved mobile beds. Advances in Water Resources, 72, 32 - 44. 


\section{Tables}

Table 1: Collisional force equations and parameter settings [Derksen 2019]; all parameters in lattice units

\begin{tabular}{|c|c|}
\hline normal elastic force ${ }^{a}$ & $\mathbf{F}_{\mathbf{i j}}^{\mathrm{el}}=k\left(\delta_{0}-\delta\right) \frac{\left|\delta_{\lambda}-\lambda\right|}{\lambda} \frac{\left(\mathbf{n}_{\mathbf{j}}-\mathbf{n}_{\mathbf{i}}\right)}{\left|\mathbf{n}_{\mathbf{j}}-\mathbf{n}_{\mathbf{i}}\right|}$ if $\delta<\delta_{0}$ and $\left|\delta_{\lambda}\right|<\lambda ; \quad \mathbf{F}_{\mathbf{i j}}^{\mathrm{el}}=\mathbf{0}$ otherwise \\
\hline & $k=5.0 ; \delta_{0}=0.50 ; \lambda=0.50$ \\
\hline \multirow[t]{2}{*}{ normal lubrication force ${ }^{b}$} & $\mathbf{F}_{\mathbf{i} \mathbf{j}}^{\mathbf{n}}=k^{n}\left(\frac{1}{\delta^{*}}-\frac{1}{\delta_{d}}\right) \frac{\left|\delta_{\lambda}-\lambda\right|}{\lambda} \Delta \mathbf{u}^{\mathbf{n}}$ if $\delta^{*}<\delta_{d}$ and $\left|\delta_{\lambda}\right|<\lambda ; \quad \mathbf{F}_{\mathbf{i j}}^{\mathbf{n}}=\mathbf{0}$ otherwise \\
\hline & $k^{n}=5.0 ; \delta_{d}=1.0 ; \delta_{\text {sat }}=0.20$ \\
\hline \multirow[t]{2}{*}{ tangential lubrication force ${ }^{c}$} & $\mathbf{F}_{\mathbf{i j}}^{\mathbf{t}}=k^{t}\left(\frac{1}{\delta^{*}}-\frac{1}{\delta_{d}}\right) \frac{\left|\delta_{\lambda}-\lambda\right|}{\lambda} \Delta \mathbf{u}^{\mathbf{t}}$ if $\delta^{*}<\delta_{d}$ and $\left|\delta_{\lambda}\right|<\lambda ; \quad \mathbf{F}_{\mathbf{i j}}^{\mathbf{t}}=\mathbf{0}$ otherwise \\
\hline & $k^{t}=0.50$ \\
\hline \multicolumn{2}{|c|}{$\begin{array}{l}{ }^{a} \text { the force on marker point } i \text { with normal } \mathbf{n}_{\mathbf{i}} \text { due to marker point } j \text { on a different particle with normal } \mathbf{n}_{\mathbf{j}} \text { at } \\
\text { normal distance } \delta \text { and tangential distance } \delta_{\lambda}\end{array}$} \\
\hline \multicolumn{2}{|c|}{$\begin{array}{l}{ }^{\mathrm{b}} \text { the force on point } i \text { due to point } j \text { on a different particle due to the relative velocity in normal direction } \\
\text { between the two points } \Delta \mathbf{u}^{\mathrm{n}} ; \delta^{*}=\delta \text { if } \delta>\delta_{\text {sat }} \text { and } \delta^{*}=\delta_{\text {sat }} \text { if } \delta \leq \delta_{\text {sat }}\end{array}$} \\
\hline
\end{tabular}

Table 2: Power numbers for PBT simulations as a function of particle aspect ratio.

\begin{tabular}{|l|l|l|l|}
\hline$\ell / d$ & $\mathrm{Po}_{f}$ & $\mathrm{Po}_{c}$ & $\mathrm{Po}_{\text {total }}$ \\
\hline 1 & 2.24 & 0.286 & 2.52 \\
\hline 2 & 2.37 & 0.537 & 2.91 \\
\hline 4 & 2.93 & 0.965 & 3.89 \\
\hline
\end{tabular}


Figures

Figure 1. Flow configurations and Cartesian coordinate system. Left: top and side view of the rectangular tank equipped with the disk. Right: top and side view of the pitched-blade turbine (PBT) that is mounted on the same shaft at the same location as the disk. The PBT rotates such that it pumps liquid in the downward (negative $z$ ) direction.
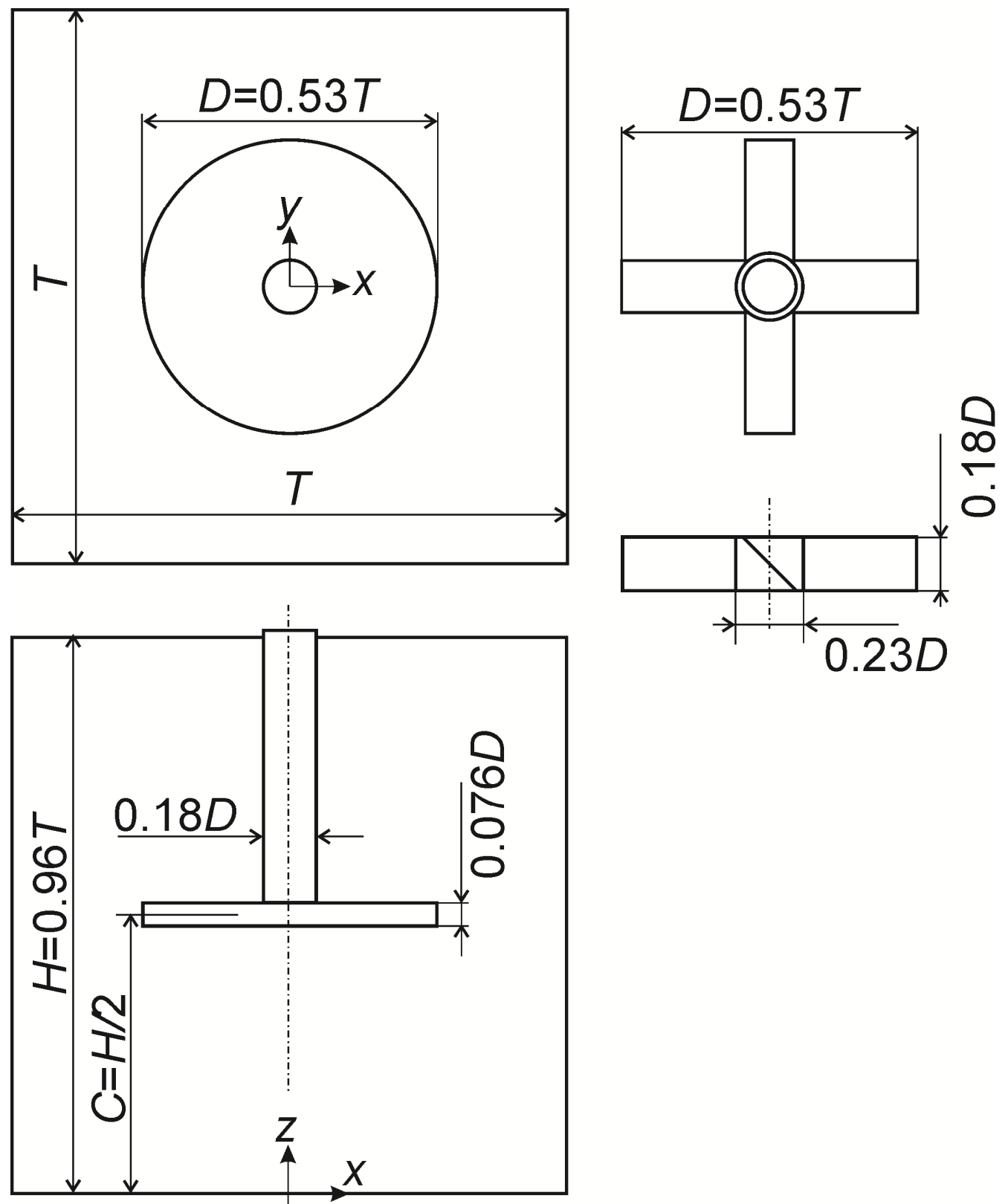
Figure 2. Particle configurations at the start of an agitated flow simulation. From left to right cylinders with aspect ratios $\ell / d=4,2$ and 1 respectively. In all cases the overall solids volume fraction is $\langle\phi\rangle=0.148$.
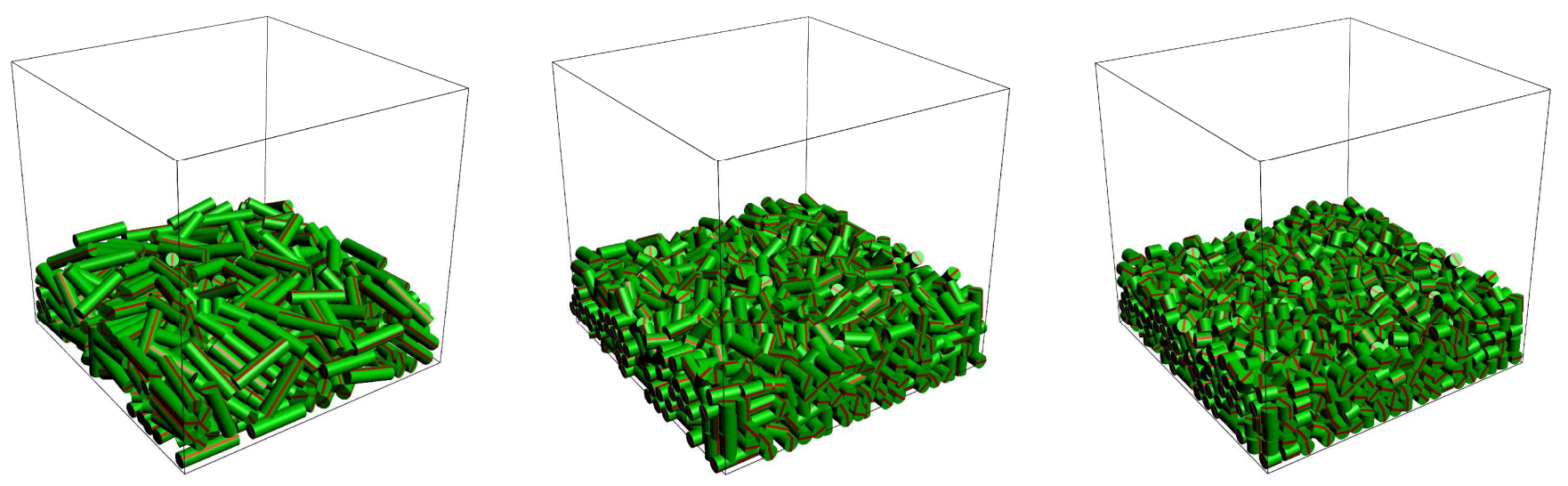
Figure 3. Instantaneous realizations of the solid-liquid flow driven by the spinning disk. Top and bottom: $\ell / d=4$ and 1 respectively. Left and right: 10 and 90 disk revolutions after startup. The color contours in the mid plane denote the liquid velocity magnitude scaled with the impeller tip speed $\mathrm{v}_{t i p}=\pi N D$.
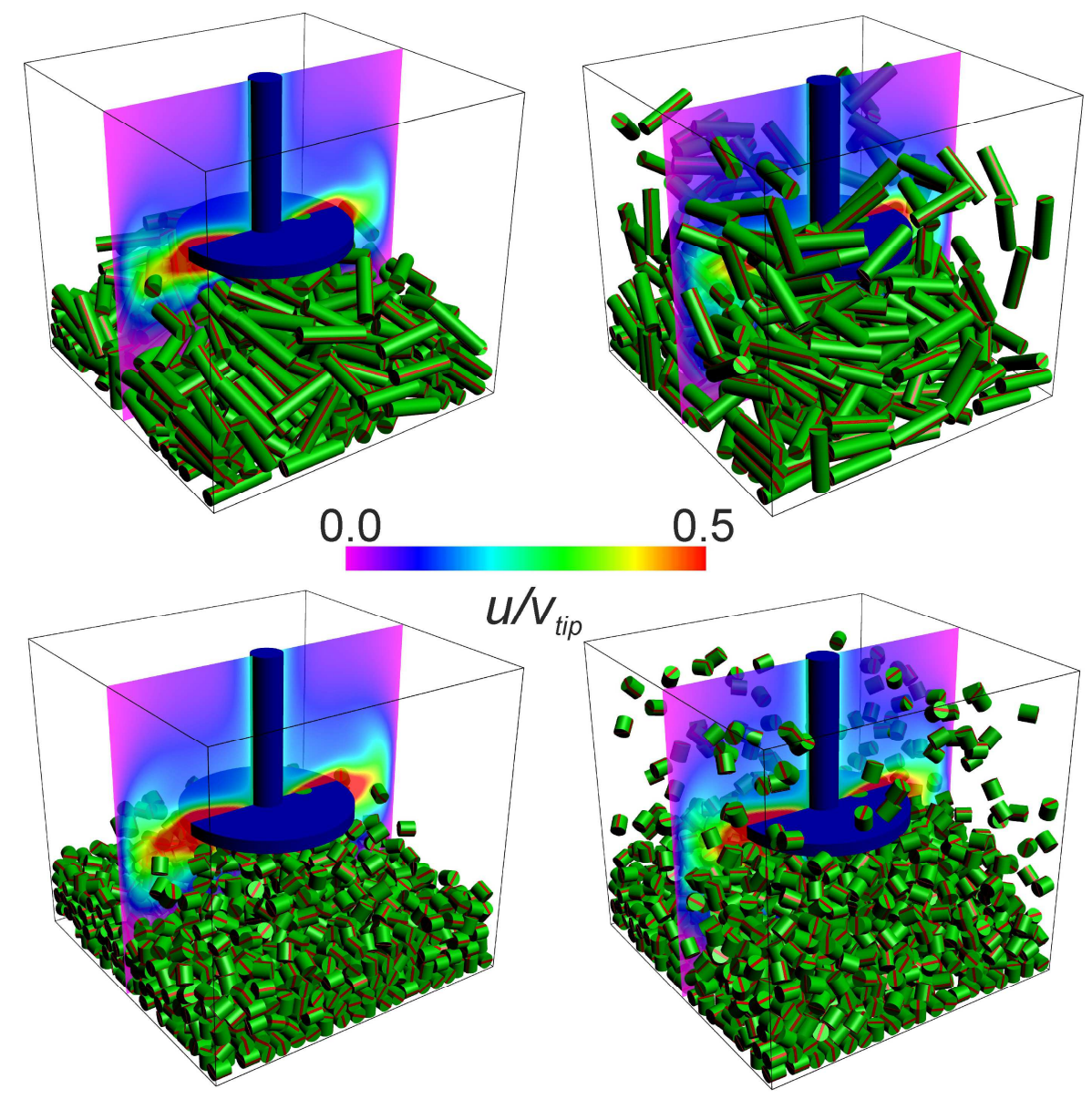
Figure 4. Instantaneous realizations of the solid-liquid flow driven by the pitched-blade turbine. Top and bottom: $\ell / d=4$ and 1 respectively. Left and right: 10 and 90 disk revolutions after startup. The color contours in the mid plane denote the liquid velocity magnitude scaled with the impeller tip speed $\mathrm{v}_{\text {tip }}=\pi N D$.
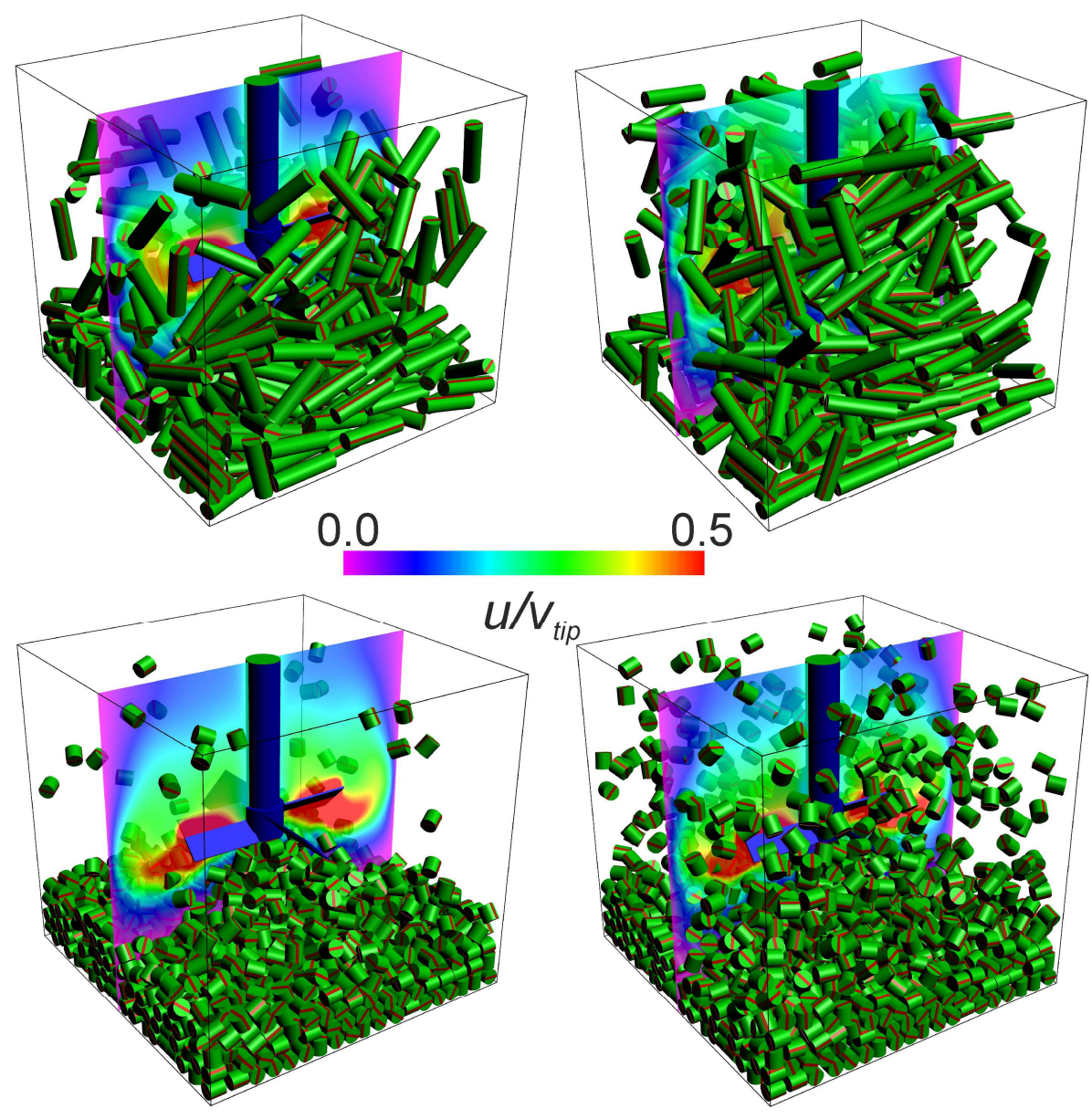
Figure 5. Time series of the average vertical center location of the particles agitated by the disk (top) and PBT (bottom); $\ell / d$ as indicated. Note the different range of the time axis of the two panels.
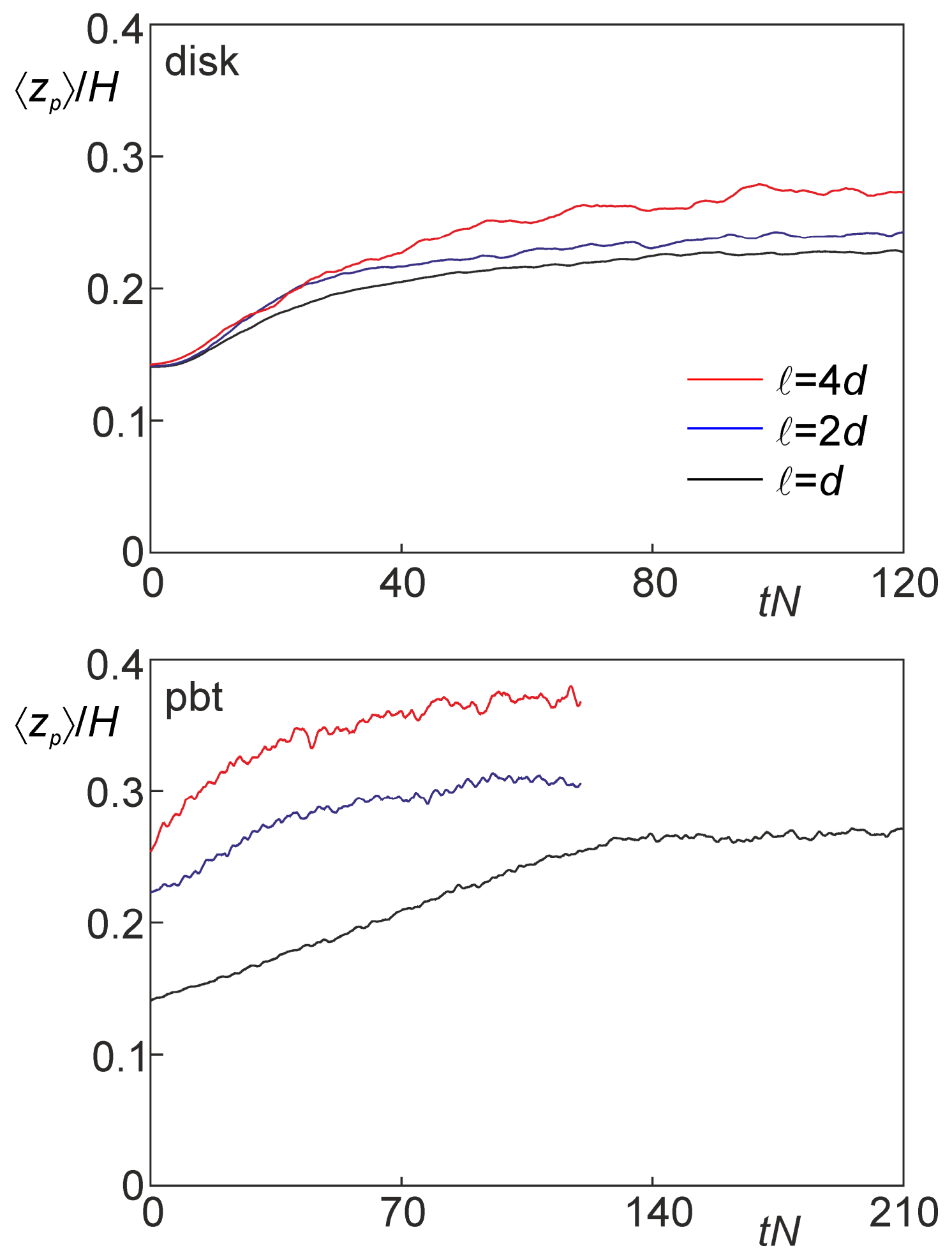
Figure 6. Time-average vertical solids concentration profile for the disk (top) and PBT system with particles of different aspect ratio $\ell / d$ as indicated.
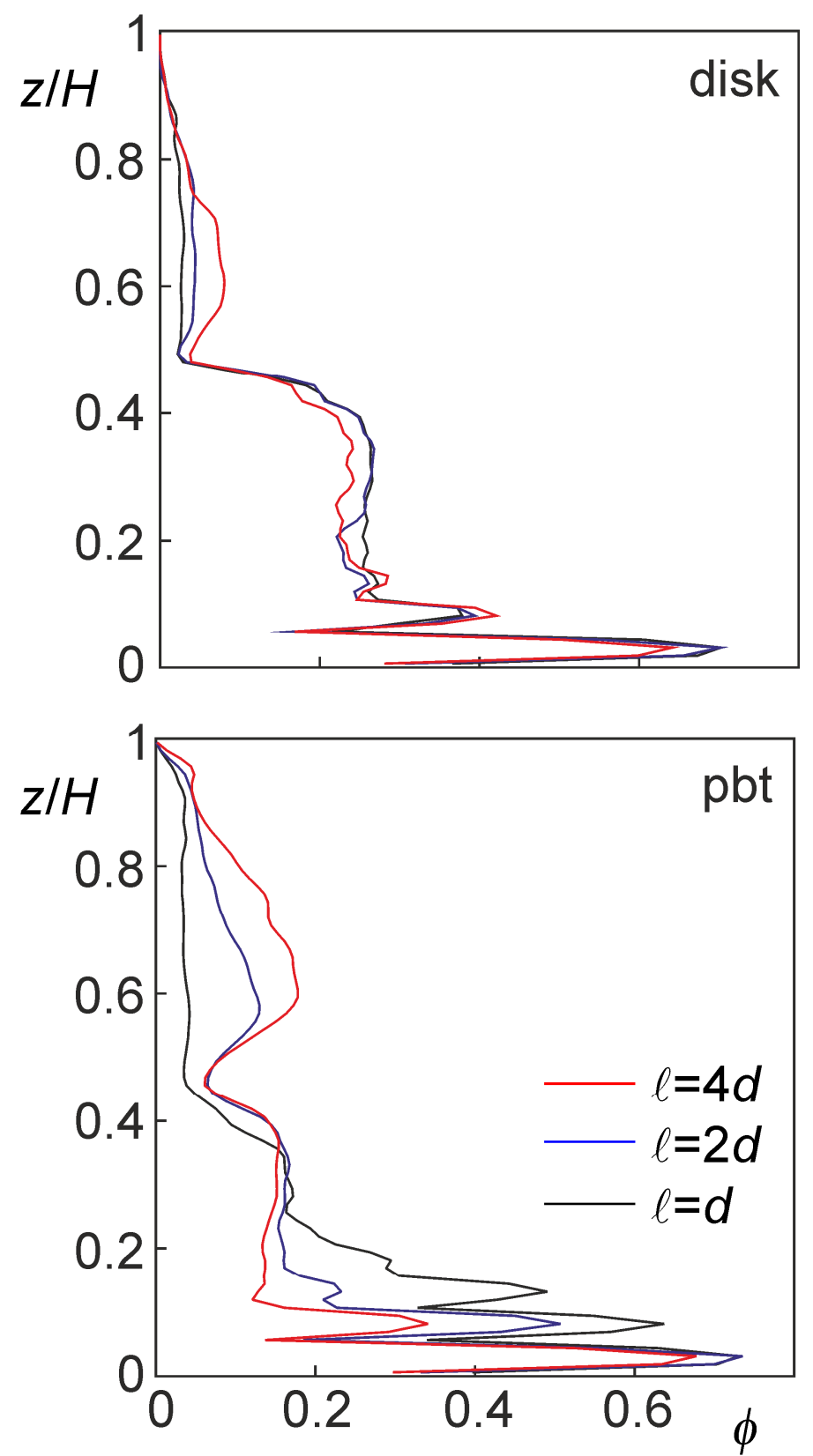
Figure 7. Vertical distribution of kinetic energy of the particles. Left: energy contained in linear motion; right: energy contained in rotational motion. Top: disk; bottom: PBT. Aspect ratio of the particles as indicated. The dimensionless quantity $k^{*}=\frac{k^{\prime}}{n m_{p} \mathrm{v}_{\text {tip }}^{2} / H}$ with $k^{\prime}$ solids kinetic energy per unit height, $m_{p}=\frac{\pi}{4} \rho_{p} \ell d^{2}$ the mass of a particle and $\mathrm{v}_{t i p}=\pi N D$ the impeller tip speed.
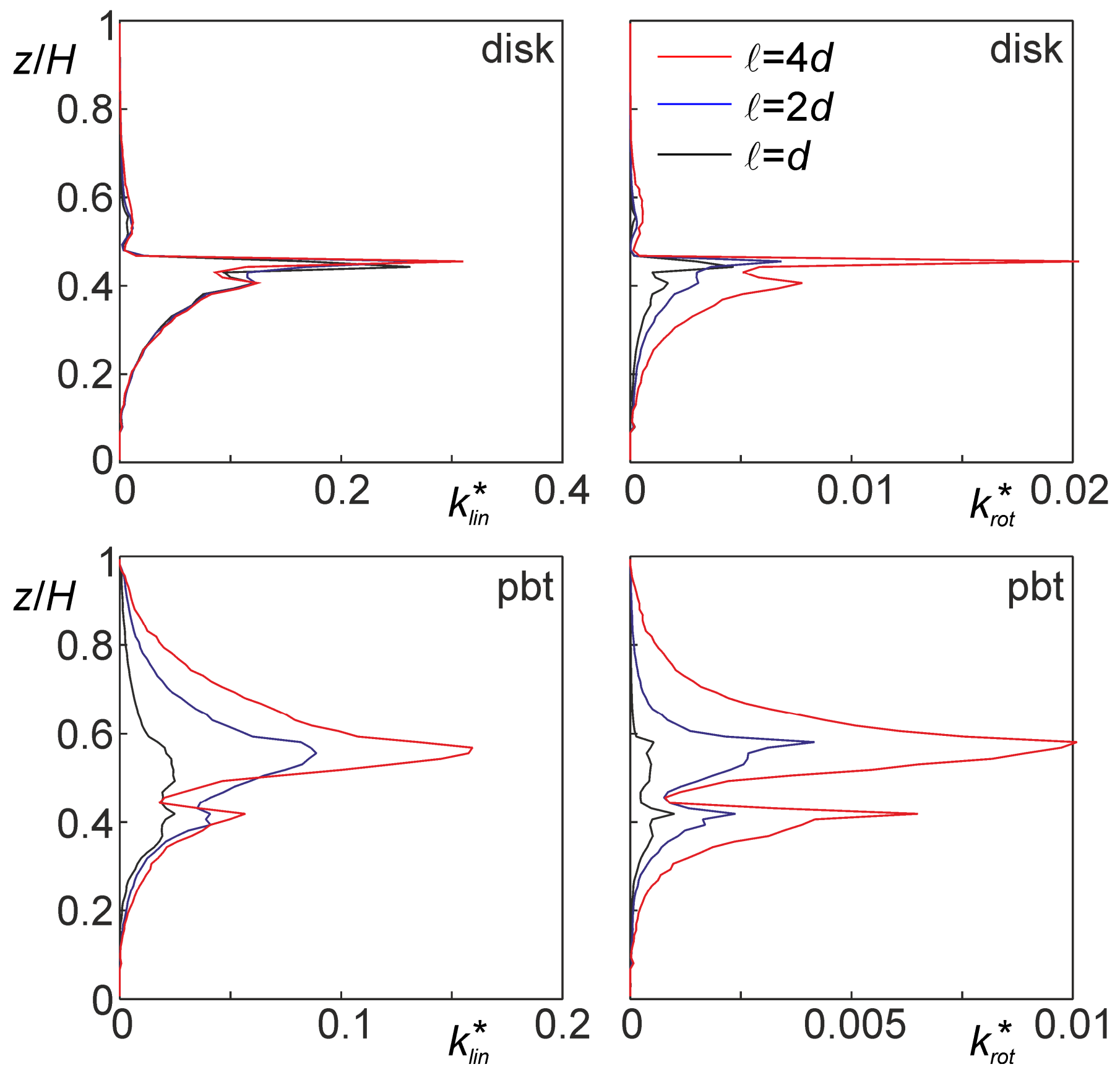
Figure 8. Orientation distribution of particles agitated by the PBT. The angle $\varphi$ is the angle between the centerline of the cylinder and the vertical. Left $\ell / d=4$; right $\ell / d=1$. We distinguish between all particles ("all") and particles with a vertical center location $z_{p}>0.2 H$. For reference $\sin \varphi$ has been included.
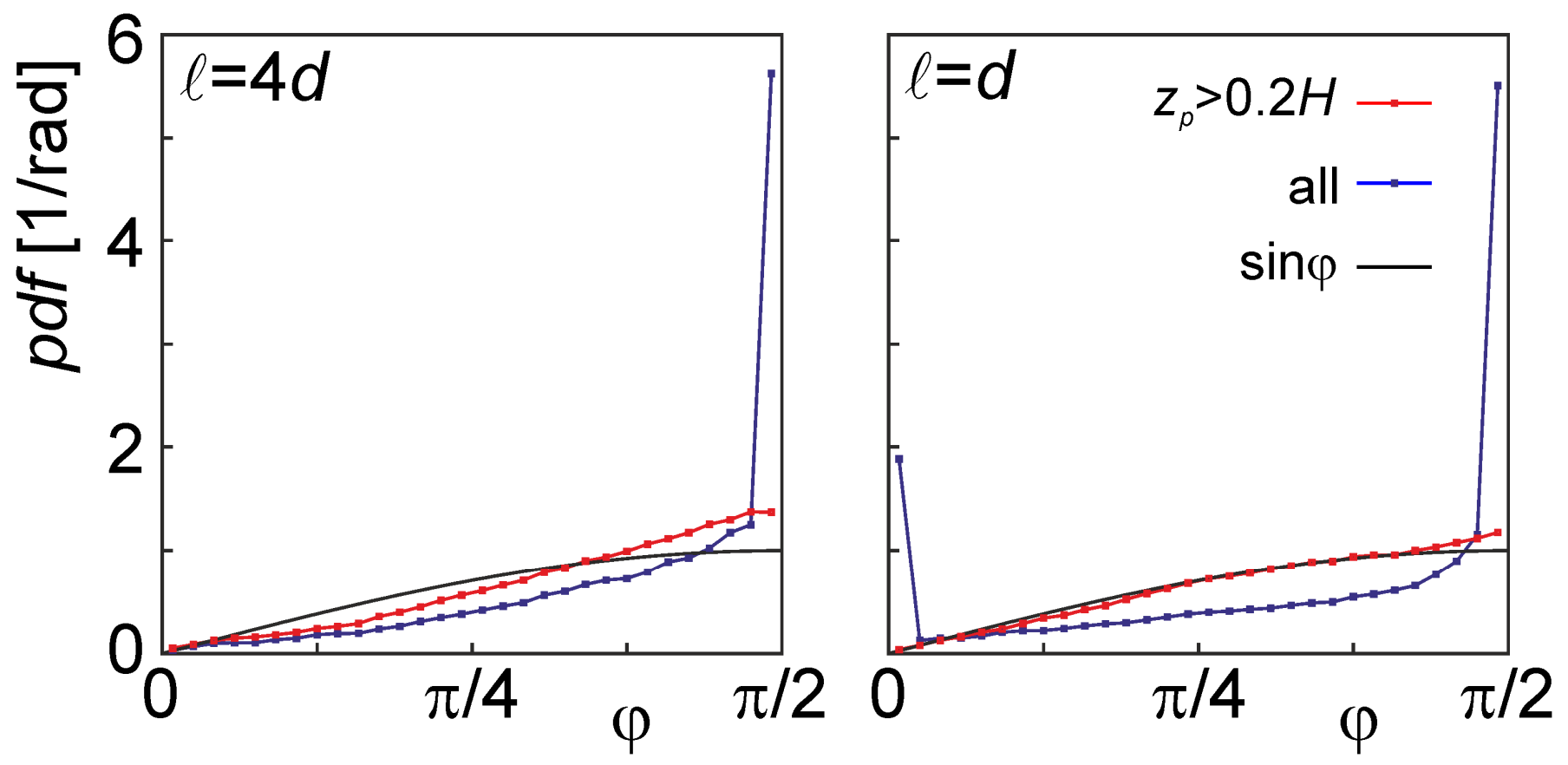
Figure 9. Orientation distribution of mobile particles $\left(z_{p}>0.2 H\right)$ agitated by the disk (top) and PBT (bottom). The $\sin \varphi$ curve indicates a uniformly random distribution.
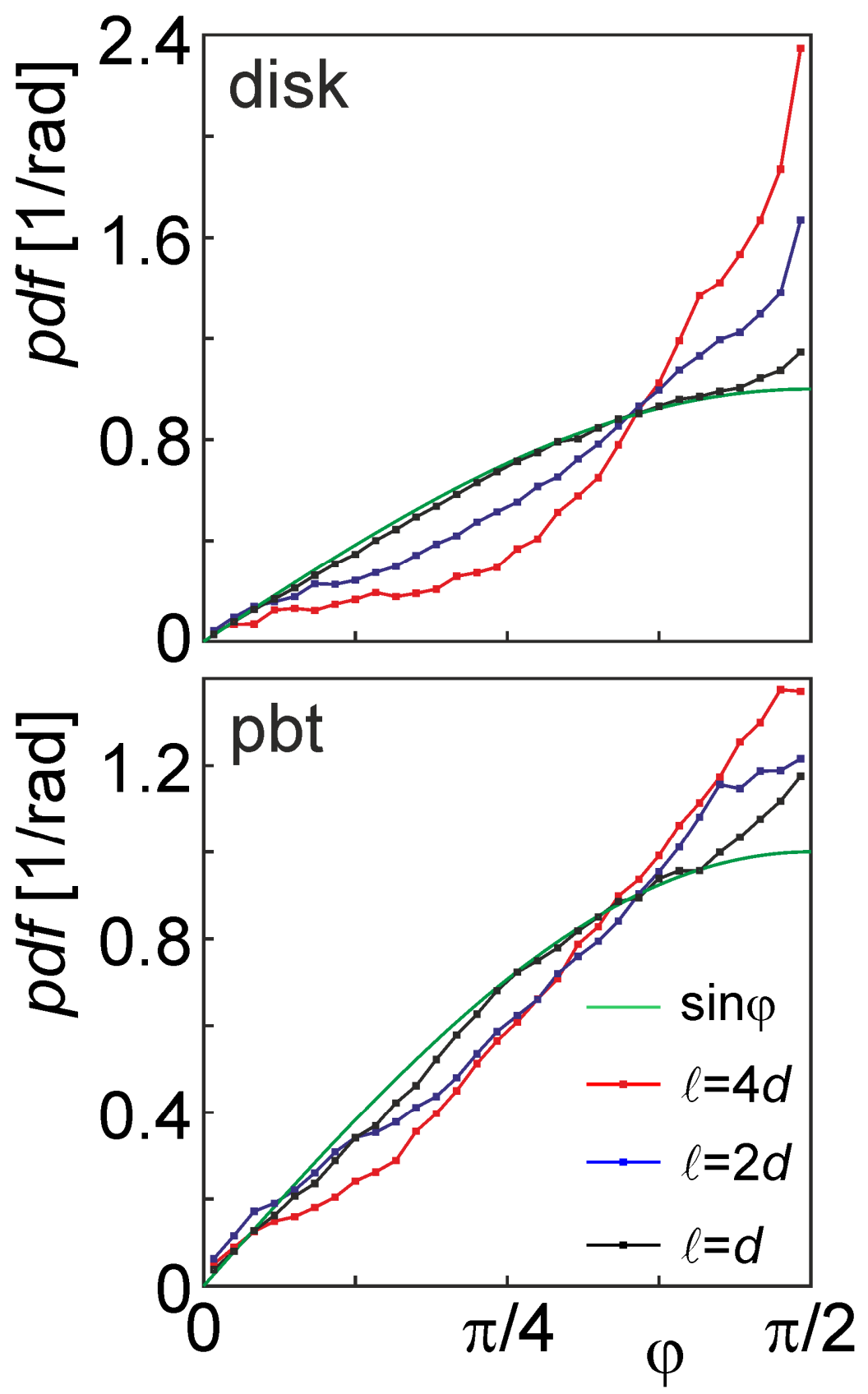
Figure 10. Time series of the power number over the last 30 impeller revolutions of PBT simulations with (from top to bottom) particles with $\ell / d=4,2$ and 1. Blue: power due to liquid; red: power due to particle-impeller collisions. The green line in the lower panel indicates the single-phase (liquid) power number.
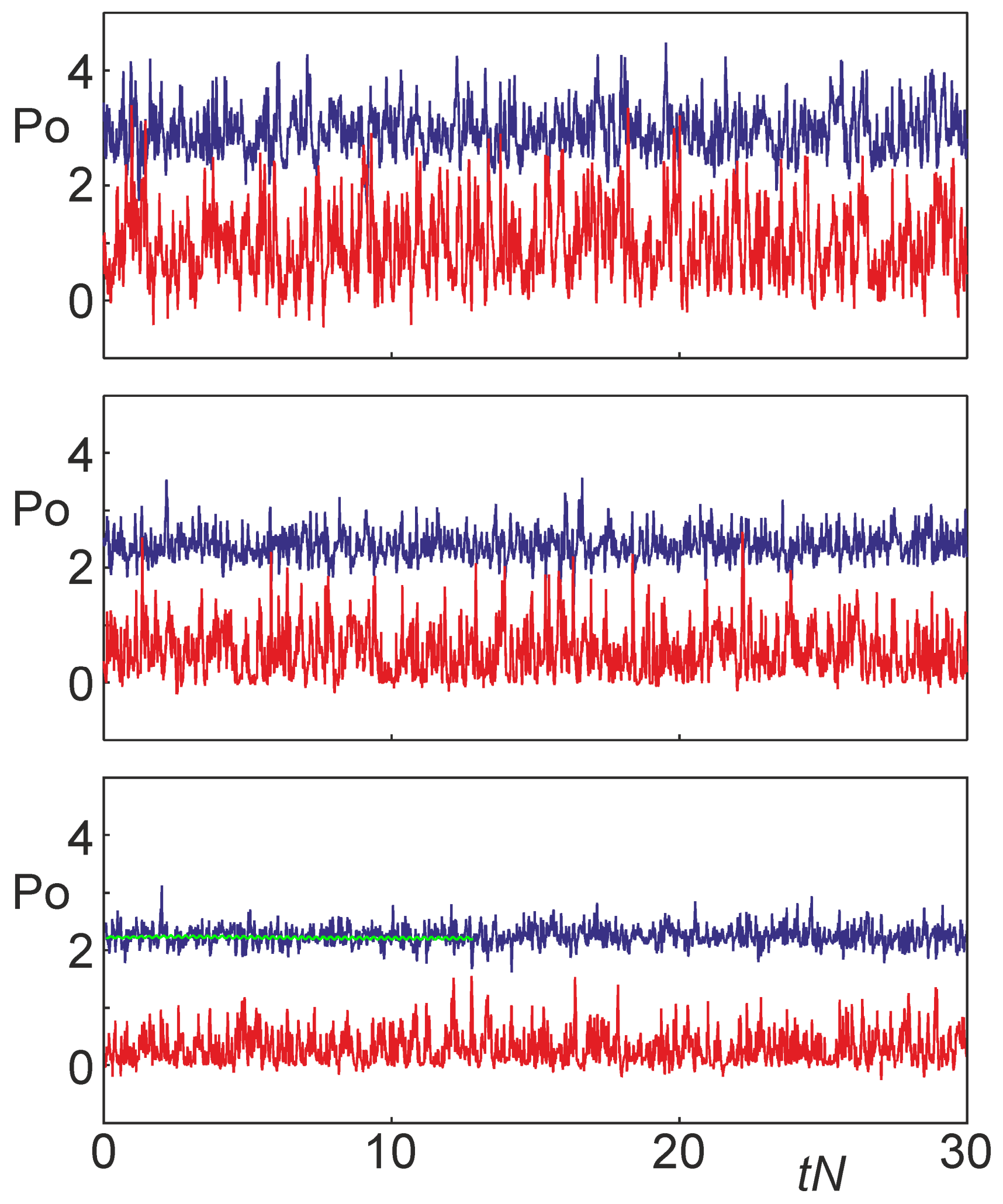